\title{
Universal Quantum Estimator
}

Artur K. Ekert, Carolina Moura Alves, f and Daniel K. L. Oi

Centre for Quantum Computation, Clarendon Laboratory, University of Oxford, Parks Road, Oxford OX1 3PU, U.K.

Michał Horodecki

Institute of Theoretical Physics and Astrophysics, University of Gdańsk, 80-952 Gdańsk, Poland.

Paweł Horodecki

Faculty of Applied Physics and Mathematics, Technical University of Gdańsk, 80-952 Gdańsk, Poland.

L. C. Kwek

Department of Natural Sciences, National Institute of Education, Nanyang Technological University, 1 Nanyang Walk, Singapore 637616

(Dated: February 1, 2008)

\begin{abstract}
We present a simple device based on the controlled-SWAP gate that performs quantum state tomography. It can also be used to determine maximum and minimum eigenvalues, expectation values of arbitrary observables, purity estimation as well as characterizing quantum channels. The advantage of this scheme is that the architecture is fixed and the task performed is determined by the input data.

PACS numbers: 03.67.Hk, 03.67.Lx
\end{abstract}

One of the the key issues in quantum information is, given an unknown quantum system, what can we learn about it. In particular, we are concerned not only with the resources needed (number of identical unknown physical systems), but also with the complexity of quantum operations required (number of different devices, networks, etc.), in order to obtain certain information about a quantum state, characterized by its density matrix $\varrho$. There are many interesting parameters of $\varrho$ we can determine, such as its maximum and minimum eigenvalues, its purity or even $\varrho$ itself (state tomography [1]), but we also can use $\varrho$ to determine expectation values of arbitrary observables or to characterize unknown quantum channels. However, this usually involves building separate devices for each task, or even building different devices for different measurements within the same task.

In this paper we present a simple, universal device, whose architecture is fixed but whose behaviour is determined by the choice of input data [2] (see also [3] for a quantum optical realization of a similar idea). In fact, with suitable input, we can directly measure all the properties mentioned before.

Consider a typical interferometric set-up for a single qubit: Hadamard gate, phase shift $\varphi$, Hadamard gate, followed by a measurement in the computational basis. Here and in the following, we borrow terminology from quantum information science and describe quantum interferometry in terms of quantum logic gates 低. We modify the interferometer by inserting a controlled- $U$ operation between the Hadamard gates, with its control on the qubit and with $U$ acting on a quantum system described by some unknown density operator $\rho$. We do

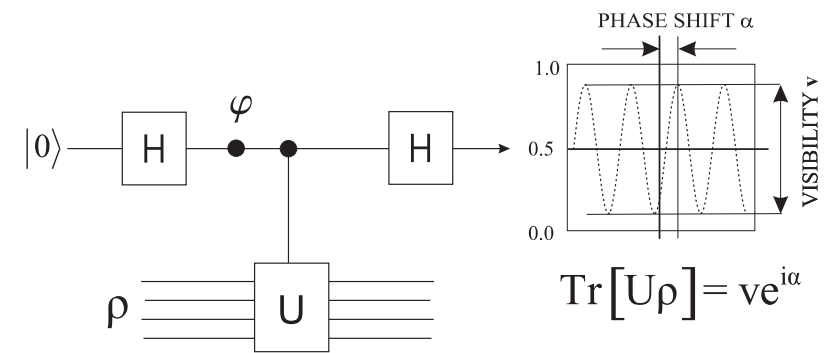

FIG. 1: Both the visibility and the shift of the interference patterns of a single qubit (top line) are affected by the controlled- $U$ operation.

not assume anything about the form of $\rho$, it can, for example, describe several entangled or separable subsystems. This set-up is shown in Fig. 11. The action of the controlled- $U$ on $\rho$ modifies the interference pattern by the factor,

$$
\operatorname{Tr} \rho U=\mathrm{v} e^{i \alpha},
$$

where $\mathrm{v}$ is the new visibility and $\alpha$ is the shift of the interference fringes, also known as the Pancharatnam phase [5].

Thus, the observed visibility gives a straightforward way of estimating the average value of unitary operators $U$ in state $\rho$ and has a variety of interesting applications. For example, it can be used to measure some entanglement witnesses $W$, as long as they are unitary operators and the corresponding controlled- $W$ operations are easy to implement [6]. Here, we focus on the applications related to quantum state state tomography. Clearly the interferometer in Fig. 1 1 can be used to estimate any $d \times d$ 
density matrix $\rho$ by a judicious choice of $d^{2}-1$ unitary operators $U$ (the basis). However, this requires building $d^{2}-1$ networks, each with a different $U$. We will show that one can build a universal state estimator i.e. a simple quantum device with a fixed architecture, where the state estimation is performed by modifying only the input data.

In order to do this, let $\rho$ to be composed of two subsystems of dimension $d, \rho=\varrho_{a} \otimes \varrho_{b}$. We will fix our controlled- $U$ to be the controlled- $V$ (SWAP) operator, such that $V|\alpha\rangle|\beta\rangle=|\beta\rangle|\alpha\rangle$ for any pure states of the subsystems (Fig. 2). Let us also introduce the maximally entangled state $\left|\phi_{+}\right\rangle=1 / \sqrt{d} \sum_{i}|i\rangle|i\rangle$ and let $P_{+}=\left|\phi_{+}\right\rangle\left\langle\phi_{+}\right|$. In this case, the modification of the interference pattern given by (11) can be written as,

$$
\mathrm{v}=\operatorname{Tr} V\left(\varrho_{a} \otimes \varrho_{b}\right)=\operatorname{Tr} \varrho_{a} \varrho_{b} .
$$

Since $\operatorname{Tr} \varrho_{a} \varrho_{b}$ is real, we can fix $\varphi=0$ and the probability of measuring the qubit to be in the state $|0\rangle$ at the output is related to the visibility by,

$$
\mathrm{v}=2 \operatorname{Pr}(|0\rangle)-1 .
$$

If $\rho$ is not separable then, writing $V=P_{+}^{T_{b}}$, we obtain $\operatorname{Tr} V \rho=\operatorname{Tr} \rho^{T_{b}} P_{+}$(the average value of partially transposed $\rho$ in the maximally entangled state $P_{+}$).

Now, let $\varrho_{b}$ be an unknown $d \times d$ density operator. Such an operator is determined by $d^{2}-1$ real parameters. In order to estimate matrix elements of $\varrho_{b}$ in a prescribed orthonormal basis, $\{|n\rangle\}$, we proceed as follows: We run the interferometer as many times as possible (limited by the number of copies of $\varrho_{b}$ at our disposal) on the input $|\psi\rangle\langle\psi| \otimes \varrho_{b}$, where $|\psi\rangle$ is a pure state of our choice. For a fixed $|\psi\rangle$, after several runs we obtain an estimation of,

$$
\mathrm{v}=\left\langle\psi\left|\varrho_{b}\right| \psi\right\rangle
$$

The diagonal elements $\left\langle n\left|\varrho_{b}\right| n\right\rangle$ can be determined using the input states $|n\rangle\langle n| \otimes \varrho_{b}$. The real part of the off-diagonal element $\left\langle n\left|\varrho_{b}\right| k\right\rangle$ can be estimated by choosing $|\psi\rangle=(|n\rangle+|k\rangle) / \sqrt{2}$, and the imaginary part by choosing $|\psi\rangle=(|n\rangle+i|k\rangle) / \sqrt{2}$. In particular, if we want to estimate the density operator of a qubit, we can choose the pure states, $|0\rangle(\operatorname{spin}+z),(|0\rangle+|1\rangle) / \sqrt{2}$ $(\operatorname{spin}+x)$ and $(|0\rangle+i|1\rangle) / \sqrt{2}(\operatorname{spin}+y)$.

We can extend the procedure above to estimate expectation values of arbitrary observables. It can be shown that the mean value of an arbitrary observable can be reduced to the estimation of a binary two-output POVM [7]. Similarly, the mean value $\langle A\rangle_{\varrho_{b}}$ of an arbitrary observable $A$ in state $\varrho_{b}$ can be measured using the setup in Fig. 2 with a suitable input $\varrho_{a}$. We shall apply the technique utilized in Refs. [8, 9]. As $A^{\prime}=\gamma \mathbb{I}+A$ is positive if $-\gamma$ is the minimum negative eigenvalue of $A$, we

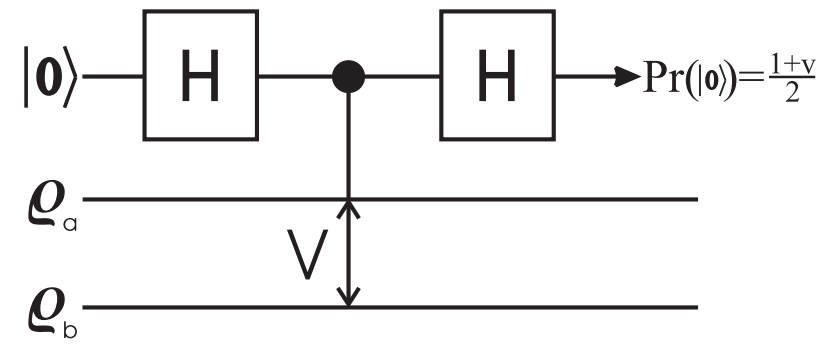

FIG. 2: Universal Quantum Estimator. By judicious choice of input $\varrho_{a}$, the state of $\varrho_{b}$ can be determined by examining the change in the visibility observed.

can construct the state $\varrho_{a}=\varrho_{A^{\prime}}=\frac{A^{\prime}}{\operatorname{Tr}\left(A^{\prime}\right)}$ and apply our interference scheme to the pair $\varrho_{A^{\prime}} \otimes \varrho_{b}$. The visibility gives us the mean value of $\mathrm{V}$ (SWAP),

$$
\mathrm{v}=\langle V\rangle_{\varrho_{A^{\prime}} \otimes \varrho_{b}}=\operatorname{Tr}\left(\varrho_{A^{\prime}} \varrho_{b}\right),
$$

which leads us to the desired value,

$$
\langle A\rangle_{\varrho_{b}} \equiv \operatorname{Tr}\left(\varrho_{b} A\right)=\mathrm{v} \operatorname{Tr} A+\gamma(\mathrm{v} d-1),
$$

where $\operatorname{Tr} \mathbb{I}=d$.

Other quantities related to $\varrho_{b}$ may be determined by simple modification of the interferometry scheme. If we have at our disposal two copies of $\varrho_{b}$ per run, by running the interferometer on the input, $\rho=\varrho_{b} \otimes \varrho_{b}$, the resulting,

$$
\mathrm{v}=\operatorname{Tr} \varrho_{b}^{2}=\sum_{i} \lambda_{i}^{2}
$$

where $\left\{\lambda_{i}\right\}$ are the eigenvalues of $\varrho_{b}$, gives us an estimate of the purity of $\varrho_{b}$. In the single qubit case, this measurement allows us to estimate various functionals of $\varrho_{b}$, such as the length of the Bloch vector,

$$
|r|=\sqrt{2 \mathrm{v}-1} .
$$

Note that the direction, however, is left completely undetermined. The procedure of estimating eigenvalues and non-linear functionals of $\varrho_{b}$ can be generalized for larger dimensional systems, but requires controlledSHIFT gates [8].

By adapting the input to the interferometer, we can also estimate the extremal eigenvalues and eigenvectors of $\varrho_{b}$. In this case, the input states are also of the form $|\psi\rangle\langle\psi| \otimes \varrho_{b}$ but we vary $|\psi\rangle$ and search for the minimum and the maximum of $\mathrm{v}=\left\langle\psi\left|\varrho_{b}\right| \psi\right\rangle$. This is usually a complicated task as it involves scanning $2(d-1)$ parameters of $\psi$. The visibility is related to the overlap of the reference state, $|\psi\rangle$ and $\varrho_{b}$ by,

$$
\begin{aligned}
\mathrm{v}_{\psi} & =\operatorname{Tr}\left(|\psi\rangle\left\langle\psi\left|\sum_{i} \lambda_{i}\right| \eta_{i}\right\rangle\left\langle\eta_{i}\right|\right) \\
& =\sum_{i} \lambda_{i}\left|\left\langle\psi \mid \eta_{i}\right\rangle\right|^{2}=\sum_{i} \lambda_{i} p_{i}
\end{aligned}
$$


where $\sum_{i} p_{i}=1$. This is a convex sum of the eigenvalues of $\varrho_{b}$ and is minimized (maximized) when $|\psi\rangle=$ $\left|\eta_{\min }\right\rangle\left(\left|\eta_{\max }\right\rangle\right)$. For any $|\psi\rangle \neq\left|\eta_{\min }\right\rangle\left(\left|\eta_{\max }\right\rangle\right)$, there exists a state, $\left|\psi^{\prime}\right\rangle$, in the neighbourhood of $|\psi\rangle$ such that $\mathrm{v}_{\psi^{\prime}}<\mathrm{v}_{\psi}\left(\mathrm{v}_{\psi^{\prime}}>\mathrm{v}_{\psi}\right)$ thus this global optimization problem can be solved using standard iterative methods, such as steepest decent [10].

Estimation of extremal eigenvalues plays a significant role in the direct detection of quantum entanglement [8] and distillation 11]. In some special cases of two qubits described by the density operator $\varrho_{b}$ such that at least one of the qubits is in the maximally mixed state, we can test for the separability of $\varrho_{b}$ by checking whether the maximal eigenvalue of $\varrho_{b}$ does not exceed $\frac{1}{2}$ (we shall return to this special case in our subsequent discussion of the quantum channel tomography).

Let us now turn our attention to characterizing quantum channels. Recall that a quantum channel is a trace preserving linear map, $\varrho \rightarrow \Lambda(\varrho)$, which takes quantum states to quantum states, and whose trivial extensions, $\mathbb{I}_{k} \otimes \Lambda$ do the same, i.e. $\Lambda$ is a completely positive map. Using the well known Jamiolkowski isomorphism [12] between quantum channels and bipartite states, quantum channels can be characterized in a simple way. The single qubit channel capacity and the distillability of entangled states can be determined by extremal eigenvalue estimation.

Suppose we have an unknown quantum channel, $\Lambda$, which we would like to characterize. The Jamiolkowski isomorphism identifies a quantum channel with its action on half of a maximally entangled state. The procedure is thus (Fig. 3): We prepare maximally entangled states of two particles $P_{+}=\frac{1}{d} \sum_{i j}|i\rangle\langle j|\otimes| i\rangle\langle j|$; We send one particle through the channel,

$$
P_{+} \rightarrow[\mathbb{I} \otimes \Lambda] P_{+}=\varrho_{\Lambda} ;
$$

We then estimate

$$
\varrho_{\Lambda}=\frac{1}{d} \sum_{i j}|i\rangle\langle j| \otimes \Lambda(|i\rangle\langle j|),
$$

which now characterizes $\Lambda$. We interpret this as $\Lambda$ mapping the $|i\rangle\left\langle\left. j\right|^{t h}\right.$-element of an input density matrix to the output matrix, $\Lambda(|i\rangle\langle j|)$. Thus, knowledge of $\varrho_{\Lambda}$ allows us to determine the action of $\Lambda$ on an arbitrary state, $\varrho \rightarrow \Lambda(\varrho)$.

Now consider the case where $\Lambda$ is a single qubit channel. The channel capacity, $Q(\Lambda)$ is an important parameter of $\Lambda$ [13, 14]. This is the optimal rate of reliably sending qubits per use of $\Lambda$. Depending on whether Alice and Bob can send classical information to each other as an additional resource, one can consider several capacities, $Q_{C}$ where $C=\varnothing, \leftarrow, \rightarrow, \leftrightarrow$, corresponding to zero

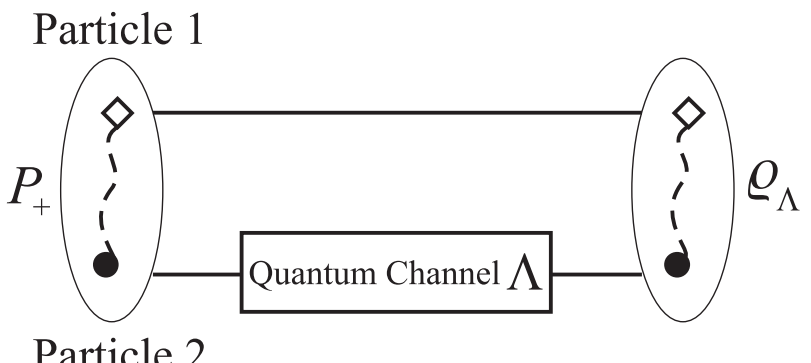

FIG. 3: A quantum channel is isomorphic with its action on half of a maximally entangled bipartite state.

way, one way and two way classical communication. In general, it is very difficult to calculate the capacity of a given channel, $\Lambda$. Here we shall provide a simple necessary and sufficient condition for a one qubit channel to have non-zero two-way capacity, $Q_{\leftrightarrow}>0$ (obviously a necessary condition for the other three capacities to be non-zero), which can be determined using our extremal eigenvalue estimation scheme.

In order to do this, we shall use the state, $\varrho_{\Lambda}$, defined in (10). Clearly, for any channel $\Lambda, \varrho_{\Lambda}$ is maximally mixed when reduced to the subsystem $A$. It is also true that for any state $\varrho_{\Lambda}$ with a maximally mixed subsystem $A$ there exists a channel $\Lambda$ which generates $\varrho_{\Lambda}$ via the formula (10). If $\varrho_{\Lambda}$ is maximally mixed when reduced to both subsystems $A$ and $B$, then the channel $\Lambda$ is called bistochastic - it maps maximally mixed states into maximally mixed states. (Two-qubit states, corresponding to bistochastic channels, have been completely characterized geometrically as tetrahedrons in $\mathbf{R}^{3}$ [15, 16]).

It is known (see 17) that a two qubit state is twoway distillable iff the operator $\varrho_{A} \otimes I-\varrho_{A B}$ has negative eigenvalue. Now for states of the type $\varrho_{\Lambda}$ (those are all states with $\varrho_{A}=\frac{I}{2}$ ), this reduces to the requirement that $\varrho_{\Lambda}$ has maximal eigenvalue greater than $\frac{1}{2}$. This is also equivalent to $Q_{\leftrightarrow}(\Lambda)>0$, since two-way distillable entanglement (which is non-zero iff given state is two way distillable) is simply the lower bound for $Q_{\leftrightarrow}(\Lambda)$ 14.

Now we can apply our estimator of the maximal eigenvalue to check whether a given one-qubit channel has a non-zero two-way capacity. Instead of fully determining the channel as in the previous section, we simply estimate the maximum eigenvalue of $\varrho_{\Lambda}$. This scheme can be also be used to find whether a given two qubit state, with one sub-system maximally entangled, is two-way distillable.

We have presented a universal quantum estimator, whose action is determined by the input. The is based on interferometry and the controlled-SWAP operation. By suitable choice of input states, we are able to perform quantum tomography, extremal eigenvalue estimation, purity tests and quantum channel characterization. Finally let us mention that the controlled-SWAP operation is a direct generalization of a Fredkin gate [18] and 
can be constructed out of simple quantum logic gates [19. This means that experimental realizations of a universal quantum estimator are within the reach of quantum technology that is currently being developed.

A.K.E. and L.C.K. would like to acknowledge the financial support provided under NSTB Grant No. 012104-0040. P.H. would like to acknowledge the support of the Polish Committee for Scientific Research and the European Commission. C.M.A. would like to acknowledge the financial support of Fundação para a Ciência e Tecnologia (Portugal). D.K.L.O would like to acknowledge the support of CESG (UK) and QAIP (contract no. IST-1999-11234).

* Electronic address: carolina.mouraalves@qubit.org

[1] K. Vogel and H. Risken Phys. Rev. A 40, 2847 (1989)

[2] M. Nielsen, I. Chuang, Phys. Rev. Lett. 79, 321 (1997)

[3] R. Filip, quant-ph/0108119

[4] M. Nielsen, I. Chuang, Quantum Computation and Quantum Information, (Cambridge University Press 2000)

[5] S. Pancharatnam. Generalized theory of interference and its applications. Proceedings of the Indian Academy of Science, XLIV(5):247-262, (1956)

[6] M. Lewenstein, B. Kraus, J. I. Cirac, P. Horodecki, Phys. Rev. A 62, art. no. 052310 (2000)

[7] P. Horodecki, unpublished

[8] P. Horodecki, A. Ekert, quant-ph/0111064

[9] P. Horodecki, quant-ph/0111036

[10] P. E. Gill,W. Murray, M. H.Wright, Practical Optimization (Academic Press, Inc., London, 1981)

[11] M. Horodecki, P. Horodecki, R. Horodecki, Phys. Rev. Lett. 78, 574 (1997); P. Horodecki, quant-ph/0111082

[12] A. Jamiolkowski, Rep. of Math. Phy. 4, 3 (1972)

[13] M. Horodecki, P. Horodecki, R. Horodecki, Phys. Rev. Lett. 85, 433 (2000)

[14] C. H. Bennett, D. P. DiVincenzo, J. A. Smolin and W. K. Wooters, Phys. Rev. A 54, 3824 (1996)

[15] M. Horodecki, R. Horodecki, Phys. Rev. A 54, 1838 (1996)

[16] D. K. L. Oi, quant-ph/0106035

[17] M. Horodecki, P. Horodecki, Phys. Rev. A 59, 4206 (1999)

[18] E. Fredkin and T. Toffoli, Int. J. Theor. Phys. 21, 219 (1982)

[19] A. Barenco, C.H. Bennett, R. Cleve, D.P. DiVincenzo, N. Margolus, P. Shor, T. Sleator, J. Smolin, and H. Weinfurter, Phys. Rev. A 52, 3457 (1995) 